\title{
A Novel Bat Algorithm for Channel Allocation to Reduce FWM Crosstalk in WDM Systems
}

\author{
Ritu \\ Department of Electronics and \\ Communication Engineering \\ Haryana Engineering College, \\ Jagadhri, India
}

\author{
Shonak Bansal \\ Department of Electronics and \\ Communication Engineering \\ PEC University of Technology, \\ Sector-12, Chandigarh, India
}

\author{
Shweta Sharma \\ Department of Electronics and \\ Communication Engineering \\ Haryana Engineering College, \\ Jagadhri, India
}

\begin{abstract}
In this paper on which work done is nature inspired algorithm named Bat algorithm. nature is good source for inspiration in life in different way. Even in many search, nature gives good example for optimization many complex problems in engineering fields. Bat algorithm is metaheuristic algorithm like particle swarm, firefly. This paper formulates on echolocation behavior to reduce the crosstalk like FWM in optical wavelength division multiplexing (WDM) system for solving channel allocation problems by using concept of OGR (Optimal Golomb ruler). The comparative study of simulation results obtained by proposed metaheuristic Bat algorithm demonstrates better and efficient generation of OGRs without the requirement of increasing total bandwidth of channel, unlike the two existing conventional algorithms i.e. Extended quadratic congruence (EQC) and Search algorithm (SA), in terms of ruler length and total channel bandwidth.
\end{abstract}

\section{General Terms}

Conventional computing, Four-wave mixing, Multi- objective, Nature-inspired, Metaheuristics Optimization.

\section{Keywords}

Channel allocation,Genetic algorithm, Metaheuristic Bat optimization algorithm, Optimal Golomb ruler, FWM (Four Wave Mixing), WDM (Wave Division Multiplexing).

\section{INTRODUCTION}

FWM interference (crosstalk) is most important source perform demotion of WDM channel system. In an attempt to reduce the FWM crosstalk in WDM system unequally spaced channel allocation (USCA) techniques have been studied in [1], [3] so that performance can be improve. In unequal channel spacing allocation techniques to make certain that no FWM signal will generated at any channel frequencies. However, USCA techniques have the drawback of increased of optical channel bandwidth requirement compared to equally spaced channel allocation (ESCA). So the frequencies between any two channel is different from any other channel in minimum

Operating bandwidth. In this paper (OGRs) optimal Golomb rulers concept used for unequally spaced bandwidth channel allocation without affecting total bandwidth to reduce FWM interference in WDM system.This USCA technique suppress the FWM crosstalk signals in the optical WDM systems without inducing additional cost in terms of channel bandwidth [6], [14]-[16].Golomb rulers drive a class of NP-complete problems [18]. Various search algorithms represent to solve Golomb ruler problem like exhaustive search [23] for higher order marks, constraint programming [21], local searches [22]. The success of ORGs problem solve by nature-inspired algorithms such as Genetic Algorithm [24]-[28], Tabu Search (TS) [27], Biogeography Based Optimization. (BBO) [28][30], Big Bang-Big Crunch (BB-BC) evolution theory [32],
[33], and Firefly Algorithm (FA) [34]. Hence, nature-inspired algorithms seem to be very effective solutions for the NPcomplete problems. In this paper, a new nature-inspired metaheuristic algorithm based on echolocation behaviours of bets namely Bat algorithm for finding the OGR sequences is being presented.

After this the paper represents the concept of Golomb rulers, Bat based channels allocation algorithm. Section 3 introduces with Bat nature-inspired optimization algorithm. Section 4 presents the problem formulation. Section 5 presents the simulation results and performance comparison of proposed algorithm and Section 6 presents the conclusion and future scope of the research.

\section{GOLOMB RULERS}

Golomb ruler refers to a set of positive integers named as marks and no distinct pairs of numbers from the set have the same difference [38]-[40]. The difference between the values of any two marks is called the distance between those marks. The difference between the largest and smallest number is referred to as the length of the ruler. The number of marks on a ruler is referred to as the size of the ruler. A perfect Golomb ruler measures all the non-negative integer distances from 0 to length $L$ of the ruler [40]-[43]. An optimal Golomb ruler is the shortest length ruler for a given mark. There can be numerous different OGRs for a specific marks value. Figure 1 show an example of 6-marks non- optimal Golomb ruler having ruler length 17. The distance associated between each pair of marks is also shown in Figure 1. As clear from Figure 1 that the distance numbers 14 and 15 are missing so it is not a perfect Golomb ruler sequence.

\section{BAT ALGORITHM}

Bat algorithm, a meta-heuristic population-based optimization algorithm based on the theories of the echolocation behavior of bats.

Bat theory uses a type of sonar called echolocation, which used to detect prey, avoid obstracles and locate their food and insects in the dark [44]. Bats release a very loud sound pulse and these pulses strike the insects (objects) comes back as echo. Pulse comes back depend on the frequency $f$ and velocity of sound in air [44]

$$
\lambda=\mathrm{v} / f
$$

For simplicity, we now use the following approximate or idealized rules:

a. All bats use echolocation to sense distance, difference between food/prey and background barriers in some magical way. 
b. Bats fly with vary velocity vi at position xi with a fixed frequency $f$ min, varying wavelength $\lambda$ and loudness A0 to search for prey. Depends on their target a wavelength (or frequency) adjust of their emitted wavelength (or frequency) adjust the pulse rate $\mathrm{r}$. Adjust the rate pf pulse emission $\mathrm{r} \in[0,1]$, depending on the proximity of their target.

c. Although the loudness varies from a large (positive) A0 to a minimum constant value Amin.

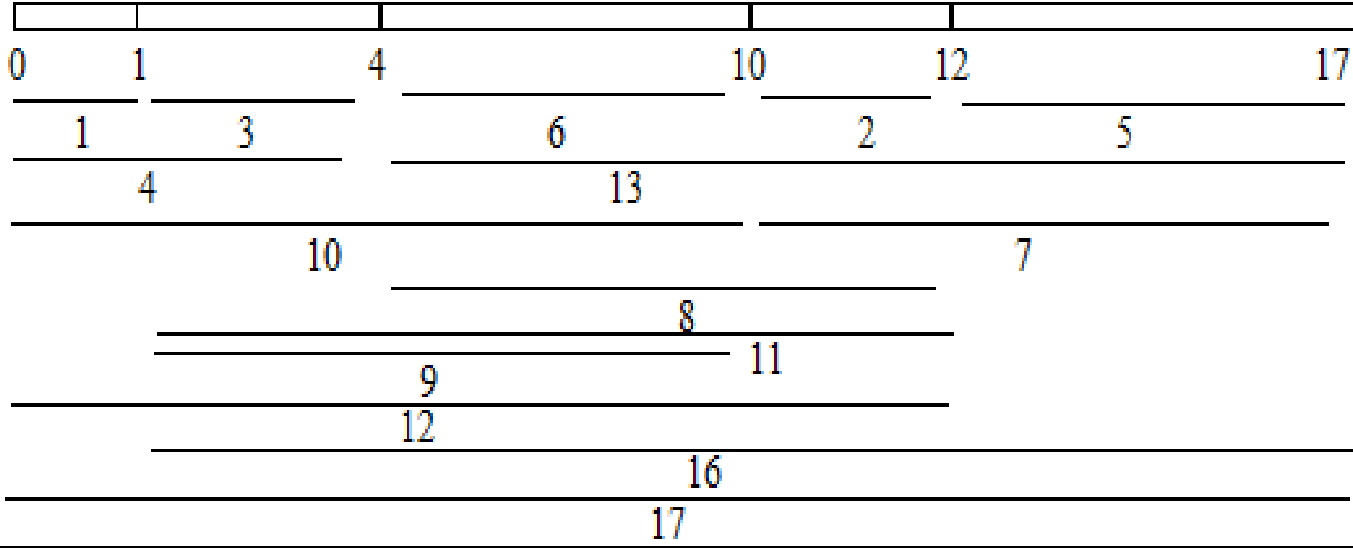

Fig1: A Non OGR having Ruler Length 17 with 6 Marks

In general the frequency lies in range $[f \min , f \max ]$.In simulations, bats positions xi and velocities vi are updated. The new solutions $x_{i}^{t}$ and velocities $v_{i}^{t}$ at time step $\mathrm{t}$ are given by [44]

$$
\begin{aligned}
& f \mathrm{i}=f \min +(f \max -f \min ) \beta, \\
& v_{i}^{t}=v_{i}^{t-1}+\left(\mathrm{x}_{i}^{t}-\mathrm{x}^{*}\right) f \mathrm{i}, \\
& x_{i}^{t}=\mathrm{x}_{i}^{t-1}+v_{i}^{t},
\end{aligned}
$$

Here $x^{*}$ is the current global best location (solution) which is located after comparing all the solutions. Where $\beta \in[0,1]$ is a random vector drawn from a uniform distribution. Initially, each bat is randomly assigned a frequency which is drawn uniformly from [ $f \min , f \max ]$.

In search part, once a solution is selected among the current best solutions, for each bat a new solution is generated locally using random process (5).

$$
X_{\text {new }}=x_{\text {old }}+\epsilon \mathrm{A}^{t}
$$

Where $\epsilon \mathrm{\epsilon}[-1,1]$ is a random number, while $\mathrm{A}^{t}=\left\langle A_{i}^{t}\right\rangle$ is the average loudness of all the bats at this time step.

Bat algorithm can be considered as a balanced combination of the standard particle swarm optimization and the intensive local search controlled by the loudness and pulse rate. As the iteration proceed the loudness the loudness $A_{i}$ and the pulse rate $r_{i}$ update as equation (6). As know loudness usually decreases once a bat has found its prey, while the pulse rate increases. For simplicity, we can also use $\mathrm{A} 0=1$ and Amin $=$ 0 , assuming Amin $=0$ means that a bat has just found the prey and temporarily stop emitting any sound. Now we have

$$
A_{i}^{t+1}=\alpha A_{i}^{t}, \mathrm{r}_{i}^{t+1}=r_{i}^{0}[1-\exp (-\gamma \mathrm{t})],
$$

Where $\gamma$ and $\alpha$ are constants. Their loudness and emission rates will be updated only if the new solutions are improved, which means that these bats are moving towards the optimal solution.

\section{PROBLEM FORMULATION}

If CS denoted the spacing between any pair of channels and the $\mathrm{n}$ is total number of channels is, then the objective is to optimize (minimize) the length of the ruler denoted as $R L$, which is given by the equation (7) [28]:

$$
R L=\sum_{i=1}^{n-1}(C S)_{i}
$$

Subject to $(C S)_{i} \neq(C S)_{j}$.

If each individual element is a Golomb ruler, the sum of all elements of an individual forms the total optical bandwidth of the channels. Thus, if an individual element is denoted as $I E$ then the second objective is to minimize the total optical bandwidth $T B W$ which is given by the equation (8):

$$
T B W=\Sigma \quad I E)_{i}
$$$$
i=1
$$

Subject to $(I E)_{i} \neq(I E)_{j}$.

Where $i, j=1,2, \ldots, n$ with $i \neq j$ are distinct in both equations (7) and (8).

The proposed pseudo-code for Bat algorithm to generate OGR sequences as unequally spaced channel-allocation in optical WDM system is shown in Figure 3 


\section{BatAlgorithm}

Begin

/* BA parameter initialization */

Define objective function $f(\mathrm{X}) ; \mathrm{X}=(x 1, \ldots, x d) T$,

Generate initial bat population $x i(i=1,2, \ldots n)$; Generate initial velocity $v i$ and Define pulse frequency $f$;

Initialize Loudness coefficient $\mathrm{A}$ and pulse rate coefficient $\mathrm{r}$;

/* End of BA parameter initialization */

Generate maximum number of iteration;

While not $T$

I*T is a termination criterion $* /$

For $t=1: \mathrm{N}$

For $i=1: \mathrm{n}$

Generate new solutions by adjusting frequency;

Updating velocities and locations/solutions [equations (2) to (4)];

If (rand > r) Select a solution among the best solutions;

Generate a local solution around the selected best solution;

End If

Generate a new solution by flying randomly;

If $\left(\right.$ rand $\left.<\mathrm{Ai} \& \mathrm{f}(\mathrm{xi})<\mathrm{f}\left(\mathrm{x}^{*}\right)\right)$

Accept the new solutions;

Update pulse rate ri (as increase) and loudness Ai (as reduce ) to improve best solutionedxx [equation (6)];

End If

End for

End for $t$

End while

Rank the bats and find the current best $\mathrm{x}$;

Postprocess results and visualization;

End

Fig 2:Pseudo-code for Algorithm

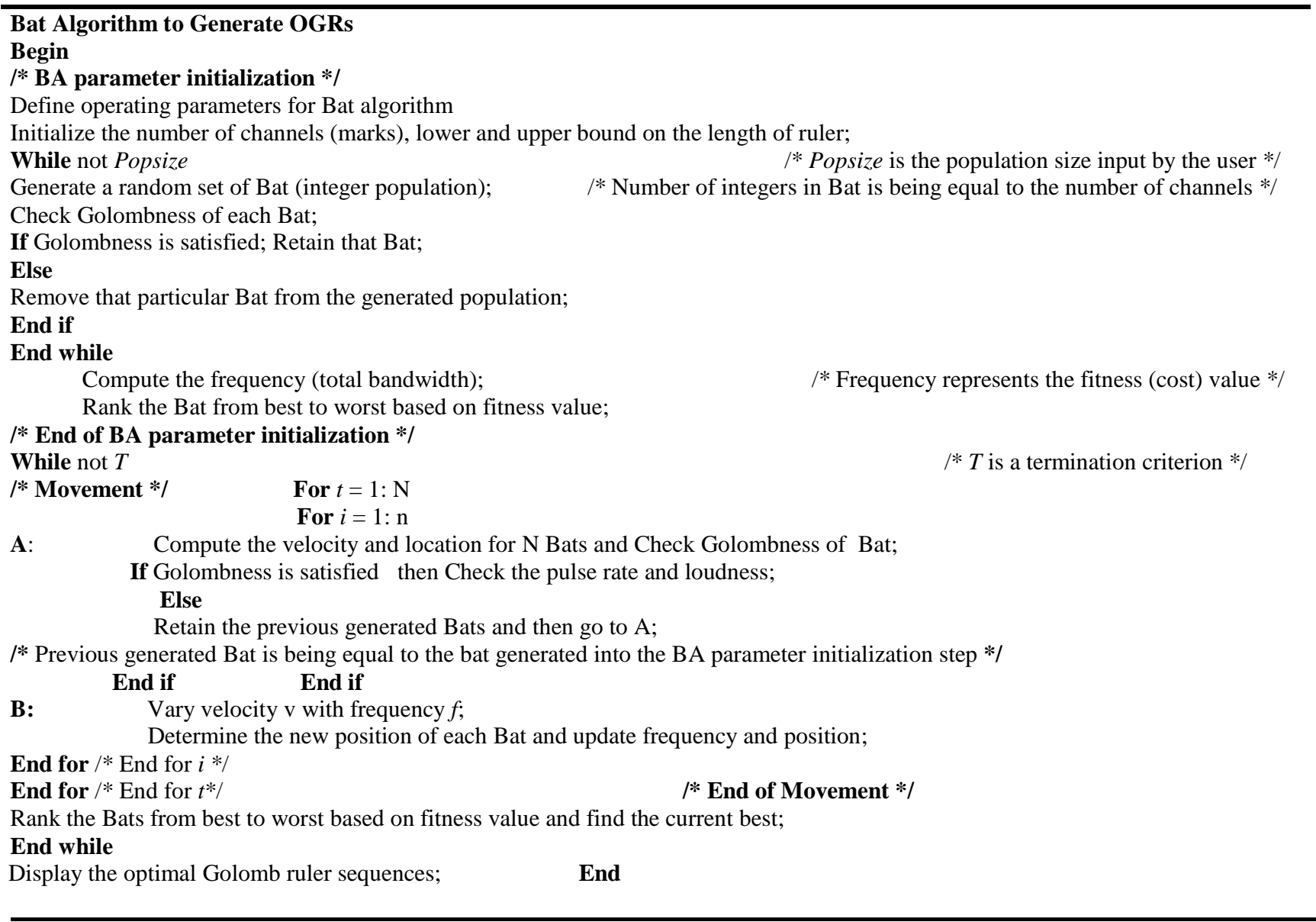




\section{SIMULATION RESULTS AND DISCUSSION}

The proposed BAT algorithm has been written and verified in Matlab-7 language [53] under Windows 7 operating system to find OGR sequences means unequal spaced channel-allocation algorithm in optical WDM systems, the proposed. This section show the effectiveness of the proposed algorithm, its performance is being compared with known OGRs [15], [20], [38]-[42], [54]-[56], EQC, SA [2], [13], [24], GA [28], and BB-BC [31], [32] algorithms of generating unequal spaced WDM channel-allocation sequences.

\subsection{Simulation Parameters for Bat Algorithm}

To get optimal OGR as optical WDM channel-allocation, after a number of careful experimentation, the optimum values of BAT parameters finally been settled in this research is reported in Table 1.

A set of 18 trials for $n=3$ to 20 are given in Table 2. With pulse rate and loudness parameters settings, the large numbers of sets of trials for various marks were conducted shown in table. Table 3 . The performance of all the sets is nearly the same as given in Table 3 .

Table 1. Simulation parameters fo Bat Algorithm

\begin{tabular}{|c|c|}
\hline Parameter & Value \\
\hline Pulse rate & 0.5 \\
\hline loudness & 0.5 \\
\hline
\end{tabular}

\subsection{Effect of Increasing Generations on Total Channel Bandwidth}

In performance of Bat algorithm iterations has great effect as shown in Table 3 i.e. ruler length and total bandwidth gets optimized after a certain numbers of iterations. Total optical bandwidth of the sequence tends to decrease as number of iterations increase; it means that the rulers reach their optimum values after a certain number of iterations. This is the point where the results are optimum and no further improvement is seen, that is, we are approaching towards the optimal solution. By carefully observation, the paper fixed the iterations of 1000 for Bat algorithm. With these parameters values, a number of sets of trials for various order marks are conducted.

\subsection{Comparison of BA with Previous Existing Algorithms in Terms of Ruler Length, Total Bandwidth and Average CPU Time}

The aim to use Bat algorithm in this paper is to optimize the length of the ruler so as to conserve the total bandwidth occupied by by the channels in less computational time. Table 2 list the length of ruler $(R L)$, total optical bandwidth $(T B W)$ and average CPU time occupied by different sequences obtained by proposed algorithm for various channels $n$ and its comparison with known OGRs [15], [20], [38]-[42], [54][56], EQC, SA [2], [13], [24], GA [28], and BB-BC [31], [32] algorithms.

EQC and SA is the conventional algorithms .The application of conventional algorithms is limited to prime powers [2], so Table 2. Show the length of ruler and total bandwidth for EQC and SA. The results obtained for 6 marks take few second to performed where as marks increase time is taken in minutes. Table 2. show that time taken by Bat algorithm is very small as compared to others non heuristic exhaustive searches. Comparing the simulation results obtained from Bat algorithm with known OGRs, EQC, SA, GA and BB-BC; it is perceived that there is a significant improvement with respect to the length of the ruler, the total bandwidth occupied and average CPU time that is, the results gets better. Figure 4 (a) and 4 (b) illustrates the graphical comparison of Bat algorithm to generate OGR sequences for optical WDM system with existing algorithms in terms of the length of the ruler and total optical bandwidth occupied by the various order mark values respectively, whereas Figure 5 illustrates the comparison of proposed Bat algorithm with $\mathrm{GA}$ and $\mathrm{BB}-\mathrm{BC}$ algorithm in terms of average CPU time (in Sec.) for various order marks. So, it is concluded from Table 2, Figures 4 and 5 that the performance of proposed Bat algorithm is better than the existing algorithms.

\section{CONCLUSION}

This paper presented the aaplication of nature-inspired metaheuristic Bat optimization algorithm to search OGRs sequences needed for optical WDM systems. BA generate very efficiently the optimal Golomb ruler's sequence that provides the unequal channel-allocation in optical WDM systems to reduce the FWM crosstalk. The performance is being compared with the existing conventional i.e EQC and SA and nature- inspired algorithms in terms of the length of ruler, total optical channel bandwidth and average CPU time obtained by the different sequences.

The preliminary results indicate that proposed Bat algorithm appears to be most efficient algorithm to generate OGRs for optical WDM systems and outperforms the existing algorithms. As to see the complexity of realizing the unequal channel spacing, so real researches of algorithms does not show. In the future, in order for these algorithms to be of practical use, it is desired that the performance of the algorithms for higher order OGRs channels may be evaluated and may be used to provide unequal channel spacing in real optical WDM systems. Though this process will be very time consuming yet this needs be done for this work to be of some use in the field of communication engineering.

\section{REFERENCES}

[1] Kwong, W. C., and Yang, G. C. 1997. An Algebraic Approach to the Unequal-Spaced Channel-Allocation Problem in WDM Lightwave Systems, IEEE Transactions on Communications, Vol. 45, No.3, pp. 352-359.

[2] Saaid, N. M. 2010. Nonlinear Optical Effects Suppression Methods in WDM Systems with EDFAs: A Review, In Proceedings of the International Conference on Computer and Communication Engineering (ICCCE), Kuala Lumpur, Malaysia.

[3] Aggarwal, G. P. 2001. Nonlinear Fiber Optics, Edition, Academic Press, San Diego, CA.

[4] Thing, V. L. L., Shum, P., and Rao, M. K. (2004). Bandwidth-Efficient WDM Channel Allocation for FourWave Mixing-Effect Minimization, IEEE Transactions on Communications, Vol. 52, No. 12, pp. $2184-2189$.

[5] Forghieri, F., Tkach, R. W., Chraplyvy, A. R., and Marcuse, D. 1994. Reduction of Four-Wave Mixing Crosstalk in WDM Systems Using Unequally Spaced Channels. IEEE Photonics Technology Letters, Vol. 6, No. 6, pp. $754-756$. 
[6] Babcock, W. C. 1953. Intermodulation interference in radio systems, Bell Systems Technical Journal, pp. 6373.

[7] Sardesai, H. P. 1999. A Simple Channel Plan to Reduce Effects of Nonlinearities In Dense WDM Systems. Lasers and Electro-Optics, (23-28, May-1999), pp. 183- 184.

[8] Forghieri, F., Tkach, R. W., and Chraplyvy, A. R. 1995. WDM systems with unequally spaced channels. J. Lightwave Technol., Vol. 13, pp. 889-897.

[9] Hwang, B. and Tonguz, O. K. 1998. A Generalized Suboptimum Unequally Spaced Channel Allocation Technique - Part I: In IM/DD WDM systems. IEEE Trans. Commun., Vol. 46, pp. 1027-1037.

[10] Tonguz, O. K. and Hwang B. 1998. A Generalized Suboptimum Unequally Spaced Channel Allocation Technique - Part II: In coherent WDM systems. IEEE Trans. Commun., Vol. 46, pp. 1186-1193.

[11] Atkinson, M. D., Santoro, N., and Urrutia, J. 1986. Integer sets with distinct sums and differences and carrier frequency assignments for nonlinear repeaters. IEEE Trans. Commun., Vol. COM-34.

[12] Randhawa, R., Sohal, J. S. and Kaler, R. S. 2009. Optimum Algorithm for WDM Channel Allocation for Reducing Four-Wave Mixing Effects. Optik120, pp. 898904

[13] http://www.compunity.org/events/pastevents/ewomp204/ jaillet_krajecki_pap_ew04.pdf.

[14] Bloom, G. S. and Golomb, S.W. 1977. Applications of Numbered Undirected Graphs. In Proceedings of the IEEE, Vol. 65, No. 4, pp. 562-570.

[15] Thing, V. L. L., Rao, M. K. and Shum, P. 2003. Fractional Optimal Golomb Ruler Based WDM Channel Allocation. In Proceedings of the 8th Opto-Electronics International Journal of Computer Applications (0975 8887) Volume 85 - No 9, January 201425and Communication Conference (OECC-2003), Vol. 23, pp. 631-632.

[16] Shearer, J. B. 1998. Some New Disjoint Golomb Rulers. IEEE Transactions on Information Theory, Vol. 44, No. 7, pp. 3151-3153.

[17] http://theinf1.informatik.unijena.de/teaching/ss10/oberse minar-ss 10

[18] Robinson, J. P. 1979. Optimum Golomb Rulers. IEEE Transactions on Computers, Vol. C-28, No. 12, (December 1979), pp. 943-944.

[19] Shearer, J. B. 1990. Some New Optimum Golomb Rulers. IEEE Transactions on Information Theory. IT-36, pp. $183-184$.

[20] Galinier, P., Jaumard, B., Morales, R. and Pesant, G. 2001. A constraint-Based Approach to the Golomb Ruler Problem. In Proceeding of 3rd International workshop on integration of AI and OR techniques (CP-AI-OR 2001).

[21] Leitao, T. 2004. Evolving the Maximum Segment Length of a Golomb Ruler. Genetic and Evolutionary Computation Conference, USA

[22] Rankin, W. T. 1993. Optimal Golomb Rulers: An exhaustive parallel search implementation.
[23] M.S.thesis,DukeUniversity,Availableathttp://people.ee.d uke.edu/ wrankin/golomb/golomb.htm

[24] Shobhika. 2005. Generation of Golomb Ruler Sequences and Optimization Using Genetic Algorithm. M.Tech Thesis, Department of Electronics and Communication Engineering, Thapar Institute of Engineering and Technology, Deemed University, Patiala.

[25] Soliday, S. W., Homaifar, A. and Lebby, G. L. 1995 Genetic Algorithm Approach to the Search for Golomb Rulers. In Proceedings of the Sixth International Conference on Genetic Algorithms (ICGA-95), Morgan Kaufmann, pp. 528-535.

[26] Robinson, J. P. 2000. Genetic Search for Golomb Arrays. IEEE Transactions on Information Theory, Vol. 46, No. 3, pp. 1170-1173.

[27] Ayari, N., Luong, T. V. and Jemai, A. 2010. A Hybrid Genetic Algorithm for Golomb Ruler Problem. In Proceeding of ACS/IEEE International Conference on Computer Systems and Applications (AICCSA 2010), pp.1-4.

[28] Bansal, S., 2014. Optimal Golomb Ruler Sequence Generation for FWM Crosstalk Elimination: Soft Computing Versus Conventional Approaches. Applied Soft Computing Journal (Elsevier), Vol. 22, pp. 443-457.

[29] Bansal, S., Kumar, S., Sharma, H. and Bhalla, P. 2011. Generation of Golomb Ruler Sequences and Optimization Using Biogeography Based Optimization. In Proceedings of 5th International Multi Conference on Intelligent Systems, Sustainable, New and Renewable Energy Technology and Nanotechnology (IISN-2011), Institute of Science and Technology Klawad, Haryana, pp 282 288.

[30] Bansal, S., Kumar, S., Sharma, H. and Bhalla, P. 2011 Golomb Ruler Sequences Optimization: A BBO Approach. International Journal of Computer Science and Information Security (IJCSIS), Pittsburgh, PA, USA, Vol 9, No. 5, pp. 63-71.

[31] Kumar S., Bansal S. and Bhalla P. 2012. Optimal Golomb Ruler Sequence Generation for FWM Crosstalk Elimination: A BB-BC Approach. In Proceedings of 6th International Multi Conference on Intelligent Systems, Sustainable, New and Renewable Energy Technology and Nanotechnology (IISN-2012), Institute of Science and Technology Klawad-133105, Haryana, India, pp. 255-262.

[32] Bansal S., Kumar S. and Bhalla P. 2013. A Novel Approach to WDM Channel Allocation: Big Bang-Big Crunch Optimization. In the proceeding of Zonal Seminar on Emerging Trends in Embedded System Technologies (ETECH-2013) organized by The Institution of Electronics and Telecommunication Engineers (IETE), Chandigarh Centre, Chandigarh, pp. 80-81.

[33] Bansal, S. and Singh, K., 2014. A Novel SoftComputing Algorithm for Channel Allocation in WDM Systems. International Journal of Computer Applications (IJCA), Vol. 85, No. 9, pp. 19-26.

[34] Bansal, S., Chauhan, R. and Kumar, P., 2014. A Cuckoo Search based WDM Channel Allocation Algorithm. International Journal of Computer Applications (IJCA), Vol. 96, No. 20, pp. 6-12. 
[35] Jain, P., Bansal, S., Singh, A. K. and Gupta, N., 2015. Golomb Ruler Sequences Optimization for FWM Crosstalk Reduction: Multi-population Hybrid Flower Pollination Algorithm. Progress in Electromagnetics Research Symposium (PIERS), Prague, Czech Republic, pp. 2463-2467.

[36] Horn, J., Nafbliotis, N., and Goldberg, D. E. 1994. A Niched Pareto Genetic Algorithm for Multiobjective Optimization. Evolutionary Computation, 1994. IEEE World Congress on Computational Intelligence, Proceedings of the first IEEE Conference on, Vol. 1, pp 82-87.

[37] Yang, X.-S., Karamanoglu, M., and He., X. S. 2014. Flower Pollination Algorithm: A Novel Approach for Multi-objective Optimization. Engineering Optimization, Vol. 46, Issue 9, pp. 1222-1237, doi: 10.1080/0305215x.2013.832237.

[38] Dimitromanolakis, A. 2002. Analysis of the Golomb Ruler and the Sidon Set Problems, and Determination of Large, Near-Optimal Golomb Rulers. Master's Thesis, Department of Electronic and Computer Engineering, Technical University of Crete.

[39] Dollas, A., Rankin, W. T., and McCracken, D. 1998. A New Algorithm for Golomb Ruler Derivation and Proof of the 19 Mark Rulers. IEEE Transactions on Information Theory, Vol. 44, No. 1, pp. 379-382.

[40] Project OGR. http://www.distributed.net/OGR.

[41] Cotta, C., Dotu, I., Fernandez, Antonio J., and Hentenryck, Pascal V. 2007. Local Search-Based Hybrid Algorithms for Finding Golomb Rulers. Kluwer Academic Publishers, Boston, Vol. 12, Issue 3, pp. 263 291.

[42] http://mathworld.wolfram.com/PerfectRuler.html

[43] http://mathworld.wolfram.com/GolombRuler.html
[44] Afshar, M. H., and Motaei, I. 2011. Constrained Big Bang-Big Crunch Algorithm For Optimal Solution of Large Scale Reservoir Operation Problem, International Journal of Optimization In Civil Engineering, pp. $357-$ 375.

[45] X.-S. Yang, A New Metaheuristic Bat-Inspired Algorithm, in: Nature Inspired Cooperative Strategies for Optimization (NISCO 2010) (Eds. J. R. Gonzalez et al.), Studies in Computational Intelligence, Springer Berlin, 284, Springer, 65-74 (2010).

[46] Ahmadi, S. and Sedighizadeh, M. 2014. An Efficient Hybrid Big Bang-Big Crunch Algorithm for Reconfiguration of Distribution System for Loss Reduction, in Conference and exhibition on Electricity Distribution, Vol 14-E-aaa-0000.

[47] Erol, O. K. and Eksin, I. 2006. A New Optimization Method: Big Bang-Big Crunch. Advances in Department, IBM Research. Engineering Software, Vol.37, pp. 106111.

[48] Yesil, E. and Urbas, L. 2010. Big Bang-Big Crunch Learning Method for Fuzzy Cognitive Maps, World Academy of Science, Engineering and Technology 71, pp. 815-824.

[49] Price, K., Storn, R. and Lampinen. J. 2005. Differential Evolution-A Practical Approach to Global Optimization. Springer, Berlin, Germany.

[50] Storn, R., and Price, K. V. 1997. Differential EvolutionA Simple and Efficient Heuristic for Global Optimization Over Continuous Spaces. Journal of Global Optimization, Vol. 11, No. 4, pp. 341-359.

[51] http://in.mathworks.com/help/matlab/index.html. Shearer, J. B., 2001. Golomb Ruler Table. Mathematics

\section{APPENDIX-A}

Table 2: Performance Comparison of proposed BAT Algorithm with Known OGR, EQC, SA, GA and BB-BC in terms of Ruler Length, Total Bandwidth, and Average CPU Time

\begin{tabular}{|c|c|c|c|c|c|c|c|c|c|c|c|c|c|c|c|}
\hline \multirow{4}{*}{$n$} & \multirow{3}{*}{\multicolumn{2}{|c|}{$\begin{array}{l}\text { Known } \\
\text { OGRs } \\
{[15],[20],} \\
{[38]-[42],} \\
{[54]-[56]}\end{array}$}} & \multicolumn{13}{|c|}{ ALGORITHMS } \\
\hline & & & \multicolumn{4}{|c|}{$\begin{array}{l}\text { Conventional } \\
\text { Algorithms }\end{array}$} & \multicolumn{6}{|c|}{ Existing Nature -Inspired Algorithms } & \multicolumn{3}{|c|}{ Proposed Algorithm } \\
\hline & & & \multicolumn{2}{|c|}{$\begin{array}{c}\text { EQC } \\
{[2],[13],} \\
{[24]}\end{array}$} & \multicolumn{2}{|c|}{$\begin{array}{c}\text { SA } \\
{[2],[13],} \\
{[24]}\end{array}$} & \multicolumn{3}{|c|}{$\begin{array}{l}\text { GA } \\
{[28]}\end{array}$} & \multicolumn{3}{|c|}{$\begin{array}{c}\text { BB -BC } \\
{[31],[32]}\end{array}$} & \multicolumn{3}{|c|}{ BA } \\
\hline & $R L$ & $\begin{array}{l}T B W \\
(H z)\end{array}$ & $R L$ & $\begin{array}{l}T B W \\
(H z)\end{array}$ & $R L$ & $\begin{array}{l}\boldsymbol{T B W} \\
(\mathrm{Hz})\end{array}$ & $R L$ & $\begin{array}{c}T B W \\
(H z)\end{array}$ & $\begin{array}{l}\text { Average } \\
\text { CPU time } \\
\text { (Sec.) }\end{array}$ & $R L$ & $\begin{array}{l}T B W W \\
(H z)\end{array}$ & $\begin{array}{c}\text { Average } \\
\text { CPU time } \\
\text { (Sec.) }\end{array}$ & $R L$ & $\begin{array}{l}T B W \\
(H z)\end{array}$ & $\begin{array}{c}\text { Average } \\
\text { CPU time } \\
\text { (Sec.) }\end{array}$ \\
\hline 3 & 3 & 4 & 6 & 10 & 6 & 4 & 3 & 4 & 0.000 & 3 & 4 & 0.000 & 3 & 4 & 0.000 \\
\hline 4 & 6 & $\begin{array}{l}11 \\
25\end{array}$ & 15 & 28 & 15 & 28 & $\begin{array}{c}6 \\
7 \\
12\end{array}$ & $\begin{array}{l}11 \\
23\end{array}$ & 0.001 & $\begin{array}{c}6 \\
7 \\
11\end{array}$ & 11 & 0.000 & $\begin{array}{c}6 \\
7 \\
11\end{array}$ & 11 & 0.000 \\
\hline 5 & 11 & 28 & - & - & - & - & 13 & $\begin{array}{l}25 \\
29\end{array}$ & 0.021 & 12 & 25 & 0.009 & $\begin{array}{l}12 \\
13\end{array}$ & 25 & 0.001 \\
\hline 6 & 17 & $\begin{array}{l}44 \\
47 \\
50\end{array}$ & 45 & 140 & 20 & 60 & $\begin{array}{l}17 \\
18 \\
21\end{array}$ & $\begin{array}{l}42 \\
44 \\
45\end{array}$ & 0.780 & $\begin{array}{l}17 \\
18\end{array}$ & $\begin{array}{l}42 \\
44\end{array}$ & 0.659 & $\begin{array}{l}17 \\
18\end{array}$ & $\begin{array}{l}42 \\
44\end{array}$ & 0.0512 \\
\hline
\end{tabular}




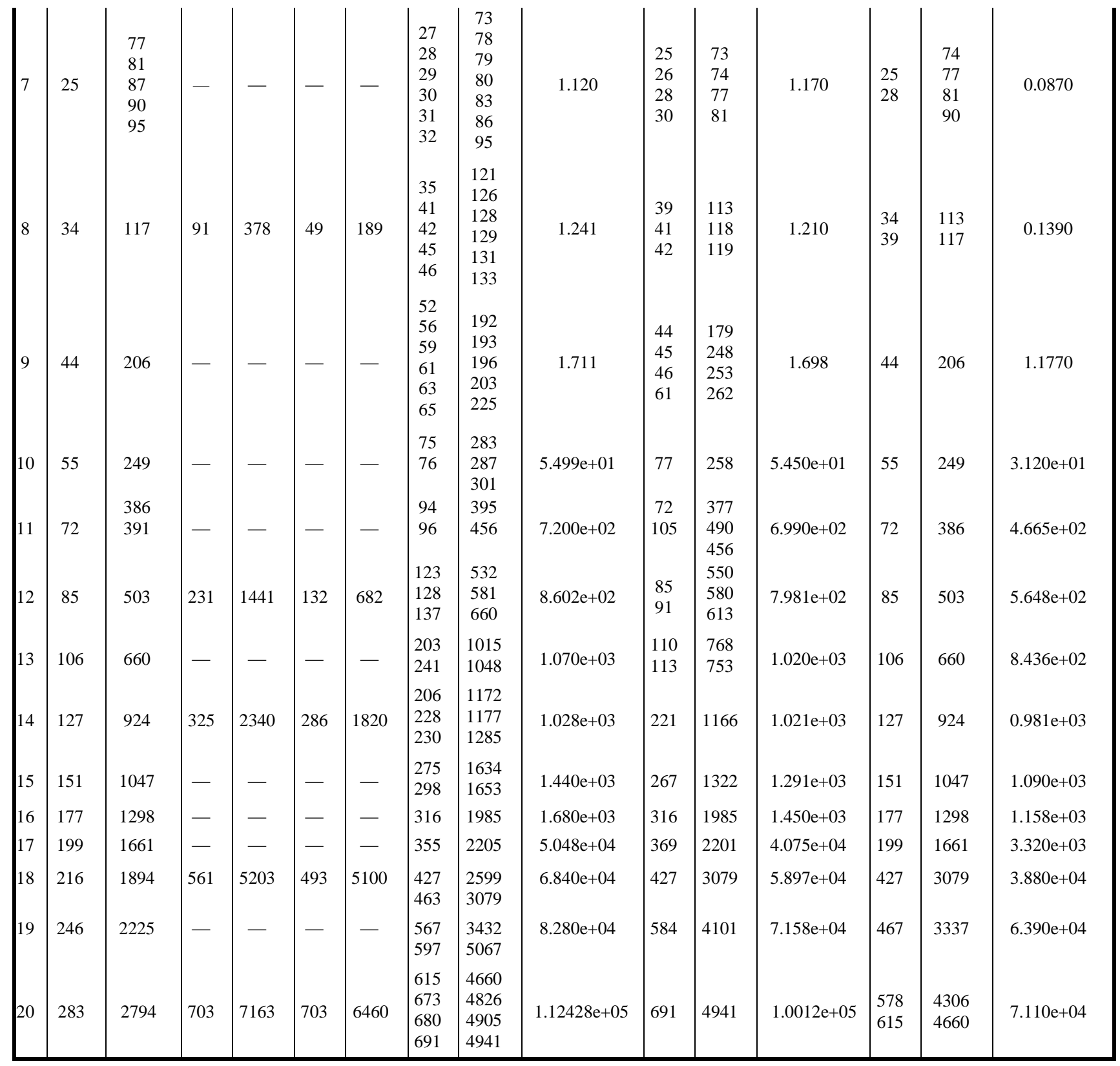


Table 3: Effect of Iterations on Total Bandwidth Generated by Proposed algorithm for Various Marks/channels

\begin{tabular}{|c|c|c|c|c|c|c|c|c|c|c|c|c|c|}
\hline \multirow{2}{*}{ Iterations } & \multicolumn{13}{|c|}{ TOTAL BANDWIDTH (Hz) } \\
\hline & $n=5$ & $n=6$ & $n=7$ & $n=8$ & $n=9$ & $n=10$ & $n=11$ & & $n=13$ & $n=14$ & $n=16$ & $n=19$ & $n=20$ \\
\hline 2 & 23 & 42 & 74 & 117 & 342 & 382 & 689 & 870 & 1156 & 2187 & 2519 & 5535 & 6435 \\
\hline 50 & 23 & 42 & 73 & 113 & 206 & 279 & 593 & 711 & 923 & 2081 & 2519 & 5535 & 6435 \\
\hline 100 & 23 & 42 & 73 & 113 & 176 & 249 & 498 & 682 & 904 & 2081 & 2484 & 5454 & 6435 \\
\hline 150 & 23 & 42 & 73 & 113 & 176 & 249 & 386 & 520 & 886 & 1937 & 2407 & 5292 & 6435 \\
\hline 200 & 23 & 42 & 73 & 113 & 176 & 249 & 386 & 503 & 823 & 1775 & 2347 & 4505 & 5361 \\
\hline 250 & 23 & 42 & 73 & 113 & 176 & 249 & 386 & 503 & 794 & 1332 & 2149 & 4458 & 5361 \\
\hline 350 & 23 & 42 & 73 & 113 & 176 & 249 & 386 & 503 & 660 & 1332 & 2149 & 4369 & 5055 \\
\hline 400 & 23 & 42 & 73 & 113 & 176 & 249 & 386 & 503 & 660 & 1219 & 1985 & 4369 & 5055 \\
\hline 500 & 23 & 42 & 73 & 113 & 176 & 249 & 386 & 503 & 660 & 1159 & 1958 & 4101 & 4941 \\
\hline 600 & 23 & 42 & 73 & 113 & 176 & 249 & 386 & 503 & 660 & 924 & 1958 & 4101 & 4859 \\
\hline 800 & 23 & 42 & 73 & 113 & 176 & 249 & 386 & 503 & 660 & 924 & 1298 & 3337 & 4660 \\
\hline 1000 & 23 & 42 & 73 & 113 & 176 & 249 & 386 & 503 & 660 & 924 & 1298 & 3337 & 4306 \\
\hline 1200 & 23 & 42 & 73 & 113 & 176 & 249 & 386 & 503 & 660 & 924 & 1298 & 3337 & 4306 \\
\hline 1500 & 23 & 42 & 73 & 113 & 176 & 249 & 386 & 503 & 660 & 924 & 1298 & 3337 & 4306 \\
\hline 1800 & 23 & 42 & 73 & 113 & 176 & 249 & 386 & 503 & 660 & 924 & 1298 & 3337 & 4306 \\
\hline 2000 & 23 & 42 & 73 & 113 & 176 & 249 & 386 & 503 & 660 & 924 & 1298 & 3337 & 4306 \\
\hline
\end{tabular}

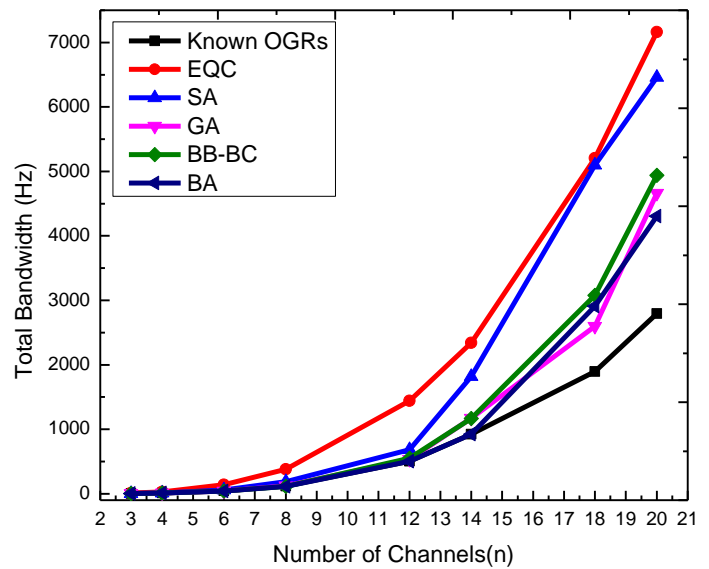

(a)

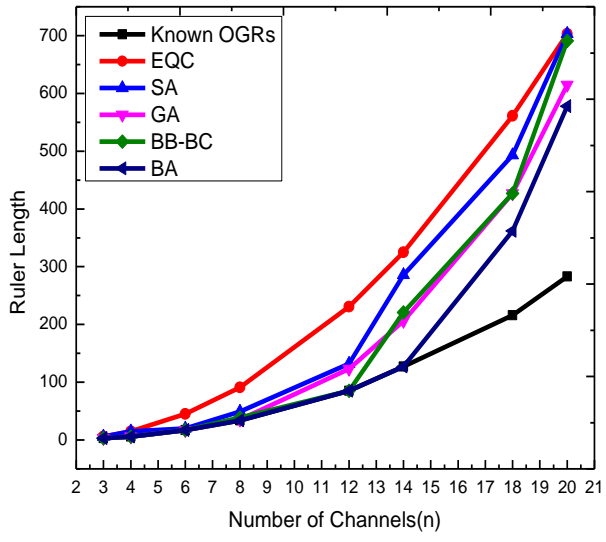

(b)

Fig 4: The propose BAT algorithm exhibits the significant reduction in (a) ) total occupied optical bandwidth and (b)ruler length in comparison to the existing algorithms i.e. Known OGR, EQC, SA, GA and BB-BC 


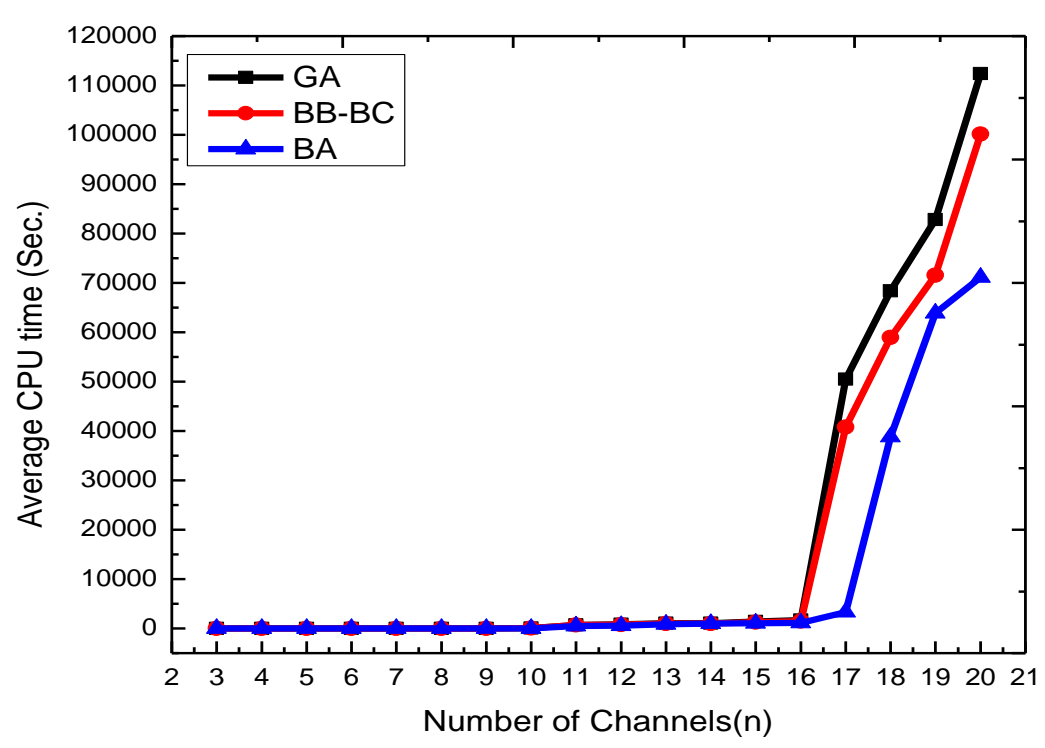

Fig 5: The proposed BAT algorithm exhibits the significant reduction in average CPU time in sec. in comparison to the existing algorithms i.e. GA and BB-BC

\section{APPENDIX-B}

The table below illustrates the optimal Golomb ruler sequences generated by the proposed BA for various marks:

Table 4. Optimal Golomb Ruler Sequences Generated By Proposed BA

\begin{tabular}{|c|c|c|}
\hline $\mathbf{n}$ & Length & Position of marks \\
\hline 3 & 3 & $\mathbf{0 1 3}$ \\
\hline \multirow[t]{2}{*}{4} & 6 & 0146 \\
\hline & 7 & 0137 \\
\hline \multirow[t]{3}{*}{5} & 11 & 0144911 \\
\hline & 12 & 013712 \\
\hline & 13 & 014613 \\
\hline \multirow[t]{2}{*}{6} & 17 & 0114101217 \\
\hline & 18 & 01381218 \\
\hline \multirow[t]{4}{*}{7} & 25 & 02310162125 \\
\hline & 26 & 0179122226 \\
\hline & 27 & 0157151827 \\
\hline & 28 & 0138122228 \\
\hline \multirow[t]{2}{*}{8} & 34 & 014915223234 \\
\hline & 39 & 013814183039 \\
\hline \multirow[t]{3}{*}{9} & 44 & 039171932394344 \\
\hline & 47 & 124111722364448 \\
\hline & 49 & 1511122033363850 \\
\hline 10 & 55 & 01610232634415355 \\
\hline \multirow[t]{2}{*}{11} & 72 & 0141328334754647072 \\
\hline & 72 & 0191924315256586972 \\
\hline
\end{tabular}




\begin{tabular}{|c|c|c|}
\hline & 103 & 13412172434495377104 \\
\hline 12 & 85 & 026242940435568757685 \\
\hline 13 & 106 & 07817213647636981101104106 \\
\hline 14 & 127 & 052838414950687592107121123127 \\
\hline 15 & 151 & 0671528405175899294121131147151 \\
\hline 16 & 177 & $\begin{array}{c}014112632566876115117134150163 \\
168177\end{array}$ \\
\hline 17 & 369 & $\begin{array}{c}2561421324954108110180190222247 \\
253337371\end{array}$ \\
\hline 18 & 445 & $\begin{array}{c}0131729357198102122147160212235 \\
256295338445\end{array}$ \\
\hline 19 & 467 & $\begin{array}{c}36252651535866104135139153243277 \\
319348402459470\end{array}$ \\
\hline 20 & 578 & $\begin{array}{c}4822274447103110118131168180319 \\
354363364405432525582\end{array}$ \\
\hline
\end{tabular}

